\title{
Numerical simulation and data analysis of the 23 July 2012 SEP event observed by ACE, STEREO-A, and STEREO-B
}

\author{
G. Qin ${ }^{1}$ and S.-Y. $\mathrm{Qi}^{2,3,4}$ \\ ${ }^{1}$ School of Science, Harbin Institute of Technology, Shenzhen 518055, PR China \\ e-mail: qingang@hit.edu.cn \\ 2 College of Sciences, Henan Agricultural University, Zhengzhou 450002, PR China \\ e-mail: qsyxiaoyi@163.com \\ 3 State Key Laboratory of Space Weather, National Space Science Center, Chinese Academy of Sciences, Beijing 100190, PR China \\ ${ }^{4}$ College of Earth Sciences, University of Chinese Academy of Sciences, Beijing 100049, PR China
}

Received 25 September 2019 / Accepted 16 March 2020

\begin{abstract}
An extremely powerful, superfast interplanetary coronal mass ejection (ICME) from the Sun on 23 July 2012 was detected by widely separated multiple spacecraft, namely STEREO-A, STEREO-B, and ACE, together with the ICME-driven shock and associated solar energetic particles (SEPs). We use the Parker spiral magnetic field model to analyze the relationship between the propagation of the shock and the SEP flux. Furthermore, we simulate the SEP event by numerically solving the three-dimensional focused transport equation of SEPs considering the shock as the moving source of energetic particles. To deal with the fact that protons and electrons behave completely differently for both parallel and perpendicular diffusion, for simplicity, we use the same diffusion model format for the simulations of protons and electrons but with different parameters. We find that the analysis can qualitatively explain the important features of the SEP flux observed by the multiple spacecraft simultaneously. In addition, the numerical results for both energetic protons and electrons approximately agree with multi-spacecraft observations.
\end{abstract}

Key words. shock waves - turbulence - scattering - Sun: coronal mass ejections (CMEs) - Sun: particle emission

\section{Introduction}

Solar energetic particles (SEPs) are generally considered to be produced from two sites: The first is the solar flare which may excite a small, short-duration, low-intensity impulsive SEP event. The second is the shock driven by the interplanetary coronal mass ejection (ICME), which may cause a large, longduration, high-intensity gradual SEP event (Reames 1995, 1999, 2013). In some events, SEPs can be observed in several locations by multiple spacecraft, connecting with the interplanetary magnetic field (IMF) to different parts of the ICME-driven shocks which cover widespread longitude and latitude ranges in space. On the other hand, strong perpendicular diffusion is considered to be the cause of such observations (e.g., Dresing et al. 2012). Therefore, it is important to analyze observational data from multiple spacecraft in order to study the acceleration and transport mechanism of energetic particles in space (Dresing et al. 2012; Wang et al. 2012; Qin \& Wang 2015; Hu et al. 2018).

On 23 July 2012, a halo superfast ICME was launched from the Sun with a maximum speed reaching $3050 \pm 260 \mathrm{~km} \mathrm{~s}^{-1}$ with the magnetic field of the ejecta reaching $109 \pm 1 \mathrm{nT}$ (Liu et al. 2014). Significant SEPs assumed to be accelerated by the ICME-driven interplanetary shock were detected during this event (Russell et al. 2013). A detailed study of the event could help to better understand the formation and evolution of SEPs and the astrophysical processes involved. Liou et al. (2014) simulated the CME-driven shock arrival time and the total magnetic field strength with a combined kinematic and magnetohydrodynamic model called H3DMHD. These latter authors found that the smaller the adiabatic index, the later the arrival time of the shock. Temmer \& Nitta (2015) measured the CME 3D kinemat- ics with the graduated cylindrical shell model, and investigated the evolution and propagation of the $\mathrm{CME}$ with the drag-based model according to the remote solar observation data available. Joyce et al. (2015) analyzed the radiation hazard of this event with the data provided by the two Solar TErrestrial RElations Observatory spacecraft (STEREO-A and STEREO-B), and Cosmic Ray Telescope for the Effects of Radiation (CRaTER). Furthermore, Zhang et al. (2016), with simulations utilizing a global MHD model, studied the geomagnetically induced currents from the sudden commencement events potentially resulting from the shock of the 23 July 2012 event. This latter work is helpful for space weather investigations.

The waves generated by streaming particles are very important for the study of SEPs because they can cause particles diffusion, and so they are included in simulations to show that wave amplification is essential for SEP transport ( $\mathrm{Ng}$ et al. 1999). In addition, realistic CME and shock geometry are key to SEP acceleration and transport, and the CME shock can distort the magnetic field lines. Therefore, realistic $\mathrm{CME}$ and shock structures from a three-dimensional MHD model were used to study the increase of SEPs (Sokolov et al. 2004; Zank et al. 2006).

Details of the origin of SEPs are also important. However, the acceleration mechanism of ICME shocks is still not fully understood. There are different ways to deal with the origin of SEPs produced by the shock. The details of the acceleration of SEPs by the shock have been investigated (e.g., Lee 1983; Gordon et al. 1999; Zuo et al. 2011, 2013; Kong et al. 2017; Qin et al. 2018). However, in other studies, the ICME shock was assumed to be a moving SEP source (Kallenrode \& Wibberenz 1997; Kallenrode 2001), so that the propagation of the particles could be focused on without considering the acceleration 
process (e.g., Heras et al. 1992, 1995; Lario et al. 1998; Wang et al. 2012; Qin et al. 2013). Solar energetic particles have also been studied by combining both acceleration and transport (e.g., Ng et al. 1999; Zank et al. 2000; Rice et al. 2003; Li et al. 2003). Based on the first-order Fermi acceleration theory, an "onion" shell model was developed to include the CMEdriven shock acceleration where the waves are excited by streaming energetic particles from shocks (Zank et al. 2000; Rice et al. 2003; Li et al. 2003).

Energetic particle diffusion is another major factor to understand the acceleration and transport of SEPs. By considering particle diffusion processes parallel and perpendicular to background magnetic field independently of one another, the standard quasi-linear theory (QLT, Jokipii 1966) of diffusion was developed. Since then, QLT has been the fundamental diffusion theory for cosmic rays, including galactic cosmic rays (GCRs) and SEPs. Although QLT works fairly well for parallel diffusion, using it for perpendicular diffusion is less straightforward. With numerical simulations, Qin et al. (2002a,b) found that nonlinear effects, that is, the interdependence between parallel and perpendicular diffusion, are significant. Matthaeus et al. (2003) developed a nonlinear guiding center (NLGC) theory for perpendicular diffusion with a parallel diffusion coefficient as the input. Furthermore, an analytic approximation of NLGC was obtained (Zank et al. 2004; Shalchi et al. 2004, 2010). In addition, Qin (2007) extended the NLGC by including the nonlinear effect in parallel diffusion with a perpendicular diffusion coefficient as the input. Later, Qin \& Zhang (2014) obtained the analytic approximation of the extended NLGC theory. On the other hand, Bieber et al. (1994) and Bieber et al. (2004) suggested that diffusion mechanisms for protons and electrons are fundamentally different. These latter authors show that the mean free path depends not only on particle rigidity but also on particle speed because of finite wave propagation speeds and dynamical representation of turbulence, and so on, and therefore the pitch angle diffusion model for protons and electrons should be different. In addition, because perpendicular diffusion depends on parallel diffusion with the nonlinear effect (Matthaeus et al. 2003), the approximated analytic form (Shalchi et al. 2004, 2010) of perpendicular diffusion should also be different for protons and electrons.

Here, we investigate the extreme 23 July 2012 SEP event observed by multi-spacecraft STEREO-A, STEREO-B, and ACE. We apply a simple and ideal Parker spiral magnetic field model to analyze the magnetic connections of the shock to the multiple spacecraft with the outward propagating CME. Furthermore, we study SEP flux by numerical simulations using the parameters observed by the spacecraft, and we compare our results with the observations. We use the numerical Shock Particle Transport Code (SPTC) by Wang et al. (2012) which considers an ICME shock to be a moving source of SEPs. In Sect. 2, we present the observations and analysis. In Sect. 3, we show the transport model. In Sect. 4, we show our simulations and the comparisons with observations. In Sect. 5, we present the conclusion and discussion.

\section{Observations and analysis}

The 23 July 2012 CME was observed launching from the Sun by the EUVI observations of the SECCHI instrument onboard the two STEREO spacecraft at about $0208 \pm 2 \mathrm{~min}$ UT (Russell et al. 2013). The source location was the sunspot group NOAA 11520 at $\mathrm{S}^{\circ} 5^{\circ} \mathrm{W} 133^{\circ}$ (Liu et al. 2014). The
CME angular width was detected as $360^{\circ} .^{1}$ The solar wind speed was typically $450 \mathrm{~km} \mathrm{~s}^{-1}$, according to the observation of STEREO-A/PLASTIC instrument (Russell et al. 2013). The fast forward shock driven by the CME was observed to reach STEREO-A at 20:55:25 UT ( $T_{\text {STA }}$ ). At 22:55 UT, the leading edge of the ICME reached STEREO-A in the ecliptic with latitude and longitude $\mathrm{S} 0.07^{\circ} \mathrm{W} 121.3^{\circ}$, and heliocentric distance $0.96 \mathrm{au}$ (Russell et al. 2013). The latitude and longitude of STEREO-B is S0.16 ${ }^{\circ}$ E115.2 ${ }^{\circ}$. The average CME transit speed was around $2000 \mathrm{~km} \mathrm{~s}^{-1}$ (Riley et al. 2016) with a height-time ${ }^{2}$ linear speed of $2003 \mathrm{~km} \mathrm{~s}^{-1}$. Later, the shock reached STEREOB at 21:21:01 UT $\left(T_{\mathrm{STB}}\right)$, but there was no shock encounter on Advanced Composition Explorer (ACE) which was $121^{\circ}$ east of STEREO-A. Simply, we assume that the shock speed is a constant $v_{\mathrm{s}}=2000 \mathrm{~km} \mathrm{~s}^{-1}$ throughout the interplanetary space in all directions.

The 23 July 2012 SEP event hs been shown to be special and complicated. Leske et al. (2015) studied it to find the bidirectional flows in ICMEs, the particles moving toward the Sun with a backside shock connected observer, and the loss-cone distributions attributable to the magnetic mirror effect. These phenomena reveal significant variations in the anisotropy of the particles. In addition, during the period from July 17 to 20, 2012, there were preceding CMEs (Bain et al. 2016; Zhu et al. 2016). Furthermore, Cohen et al. (2017) studied other SEP events to find that in some hard spectrum (HS) events the spacecraft is connected to both the preceding CMEs and the event CME, but in some soft spectrum (SS) events the spacecraft is only connected to the event CME. However, Cohen et al. (2017) suggested that during the SS 23 July 2012 event, it is not clear whether or not the observing spacecraft was connected to the preceding CMEs. Therefore, for this event, we study the anisotropy of the SEPs carefully with information from the preceding CMEs. However, it is too difficult to include the anisotropy and preceding CMEs in our analysis and modeling in this work. For the sake of simplicity, we still assume the above ideal simple model.

In order to determine the magnetic connections between the multiple spacecraft together with the shock front, we use a schematic (Fig. 1) which shows their relative positions. We use the Parker spiral model to describe the magnetic field for simplicity. Fig. 1 shows such a schematic in the ecliptic plane at 20:55 UT on 23 July, 2012. The red and blue spirals depict Parker spiral magnetic field lines passing through STEREO-A and STEREO-B at W121.3, 0.96 au, and E115.2, 1.02 au, respectively. The black spiral represents a Parker magnetic field line passing through ACE at W0, 1 au. The black straight line points to the direction of the shock nose, which was at W133 with the assumption that the shock nose is in the flare direction relative to the solar center. Therefore, the angle Shocknose-SunSTB, which is the angle between the shock nose direction and the direction from sun to the location of STEREO-B, is $112^{\circ}$. In addition, the angle Shocknose-Sun-ACE, which is the angle between the shock nose direction and the direction from sun to the location of ACE, is $133^{\circ}$. The yellow area indicates the scope of the shock sweep with width $W_{\mathrm{s}}$, the possible value of which can be deduced based on spacecraft observations. Since the shock arrived at STEREO-B without arriving at ACE according to the observations, it is assumed that half of the shock width,

1 https://cdaw.gsfc.nasa.gov/CME_list/UNIVERSAL/2012_ 07/univ2012_07.html

2 CME heights (with respect to the disk center) are measured at the fastest segment of the leading edge.

3 https://cdaw.gsfc.nasa.gov/CME_list/UNIVERSAL/2012_ 07/univ2012_07.html 


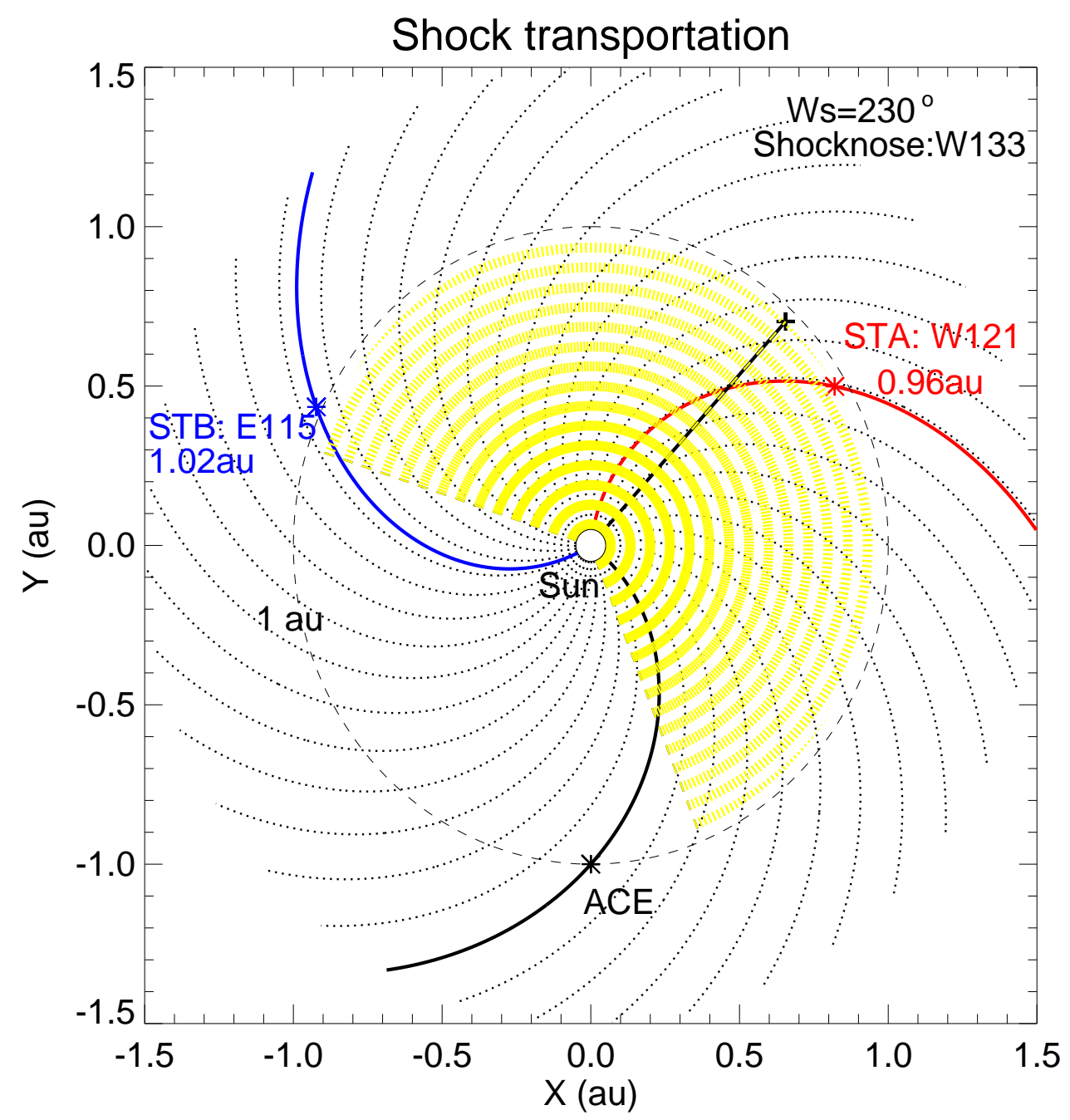

Fig. 1. Positions of the spacecraft and the shock nose direction in the ecliptic plane at 20:55 UT on 23 July 2012, the time when the shock passed STEREO-A. The red and blue spirals depict respectively magnetic field lines of Parker passing through STEREOA which was at W121.3, $0.96 \mathrm{au}$ and STEREO-B which was at E115.2, $1.02 \mathrm{au}$. The black spiral solid line represents magnetic field line of Parker passing through ACE which was at W0, $1 \mathrm{au}$. The black straight line points to the direction of shock nose, which was at $\mathrm{W} 133$. The yellow area shows the outline of the shock width $W_{\mathrm{s}}$, which is set to $230^{\circ}$.
$W_{\mathrm{s}} / 2$, should be larger than the angle Shocknose-Sun-STB, but smaller than the angle Shocknose-Sun-ACE, i.e., $112^{\circ}<W_{\mathrm{s}} / 2<$ $133^{\circ}$, or $224^{\circ}<W_{\mathrm{s}}<266^{\circ}$. In this work we choose $W_{\mathrm{s}}=230^{\circ}$, which satisfies the above inequality required by observations, as shown in Fig. 1. The Parker model of interplanetary magnetic field downstream of the shock might be incorrect.

The time profiles of proton fluxes observed by the multiple spacecraft during the 23 July 2012 SEP event are exhibited in Fig. 2. The above proton channels with similar energy are chosen for comparison. In order to investigate the transport of the CMEdriven shock and its contributions to the SEP fluxes detected by the multiple spacecraft, we divide the proton flux time profile into several parts by time with various vertical lines according to some key moments in the propagation of the shock. The dotted vertical line indicates $T 1,14: 08: 00$ UT on July 23, 2012, when the shock connection for ACE was lost. The red and blue vertical solid lines indicate the shock arrival time, 20:55 UT at STEREOA and 21:21 UT at STEREO-B, respectively, which almost coincide in Fig. 2. The dashed vertical lines indicate $T 2,15: 19: 59$ UT on July 25, when the connection of ACE was reestablished. In addition, the dot-dashed vertical lines indicate $T 3,06: 56: 00 \mathrm{UT}$ on July 27, when the connection of STEREO-B was lost again. Similarly to Fig. 2, the electron flux time profile is shown in Fig. 3. Since the parallel diffusion of energetic particles is generally much larger than their perpendicular diffusion, we only consider parallel diffusion in the remaindered of the section as a qualitative analysis.
First, we consider proton and electron SEP flux observed by STEREO-A in Figs. 2 and 3, respectively. At $T 1$, as the cobpoint of STEREO-A, which indicates the location of the shock front that is magnetically connected to the observer (Heras et al. 1995; Wang et al. 2012), is near the shock nose with the acceleration efficiency much higher than at the shock edge, the SEP flux detected by STEREO-A (the red curves in Fig. 2 for protons and Fig. 3 for electrons) was much higher than the background level. In addition, when the shock reached STEREO-A at $0.96 \mathrm{au}, T_{\mathrm{STA}}$, the SEP flux of STEREO-A almost reached the peak. This is consistent with the assumption that STEREO-A is not far away from the shock nose with strong shock acceleration, which results from the hypothesis that the shock nose is in the flare direction relative to the solar center. We note that during the shock crossing there was a significant increase of the SEP flux observations by STEREO-A. Soon after, shock reached STEREO-B at 1.02 au at $T_{\mathrm{STB}}$. Afterward, as the shock passed STEREO-A, the SEP flux detected by STEREO-A dropped dramatically.

Second, we consider proton and electron observations by STEREO-B with blue curves in Figs. 2 and 3, respectively. Solar energetic particle flux detected by STEREO-B remained at background level before $T 1$ because the shock was not wide enough to cover STEREO-B's magnetic field line (Fig. 4). However, as connection to the shock was established some time after $T 1$, the cobpoint of STEREO-B was moving toward the shock nose and the SEP flux observed by STEREO-B rose with time. We 


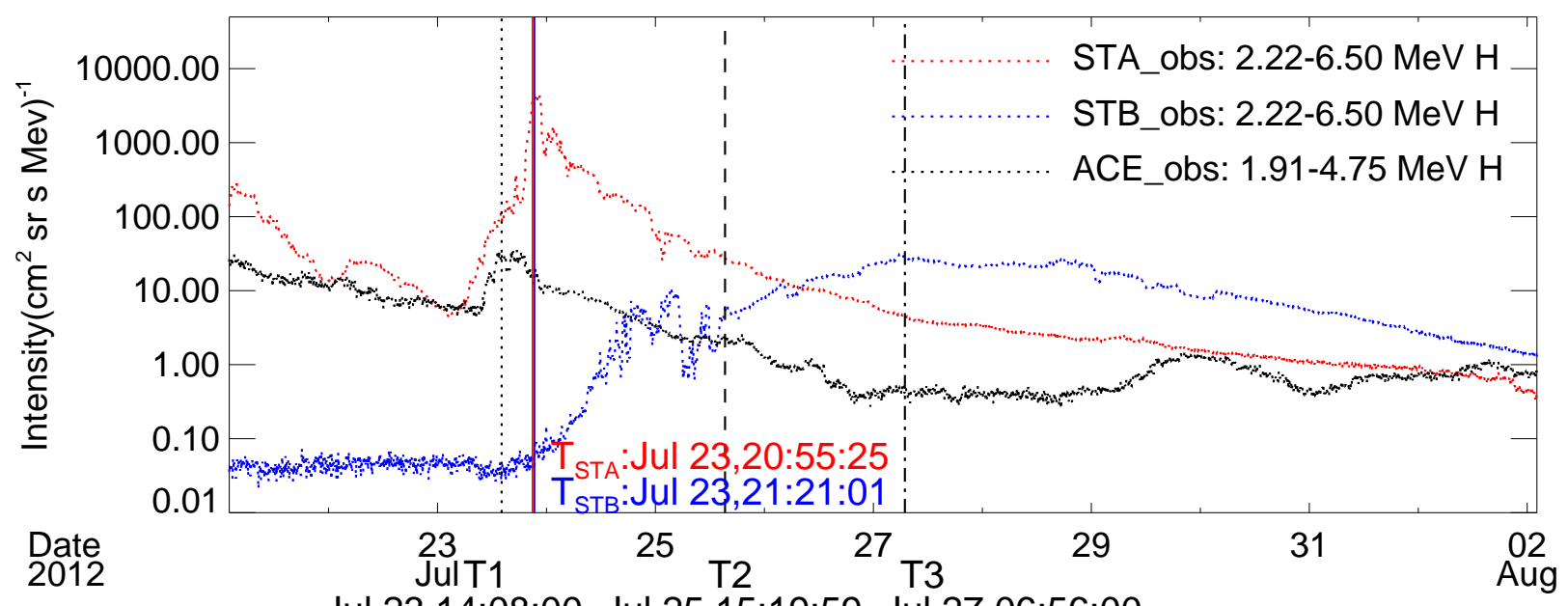

Jul 23,14:08:00 Jul 25,15:19:59 Jul 27,06:56:00

Fig. 2. Time profile of proton fluxes by the multi-spacecraft observation during the 23 July 2012 SEP event. The black, red, and blue dotted curves correspond to the observations of $1.91-4.75 \mathrm{MeV}$ protons by the EPAM instrument onboard ACE, and 2.2-6.5 MeV protons by the SEPT instruments onboard STEREO-A and STEREO-B, respectively. The red and blue vertical lines indicate the shock arrival time, 20:55 UT on STEREO-A and 21:21 UT on STEREO-B respectively. The dotted line, dashed line, and dot-dash line indicate the time $T 1$, July 23, 14:08:00, $T 2$, July 25, 15:19:59, and $T 3$, July 27, 06:56:00.

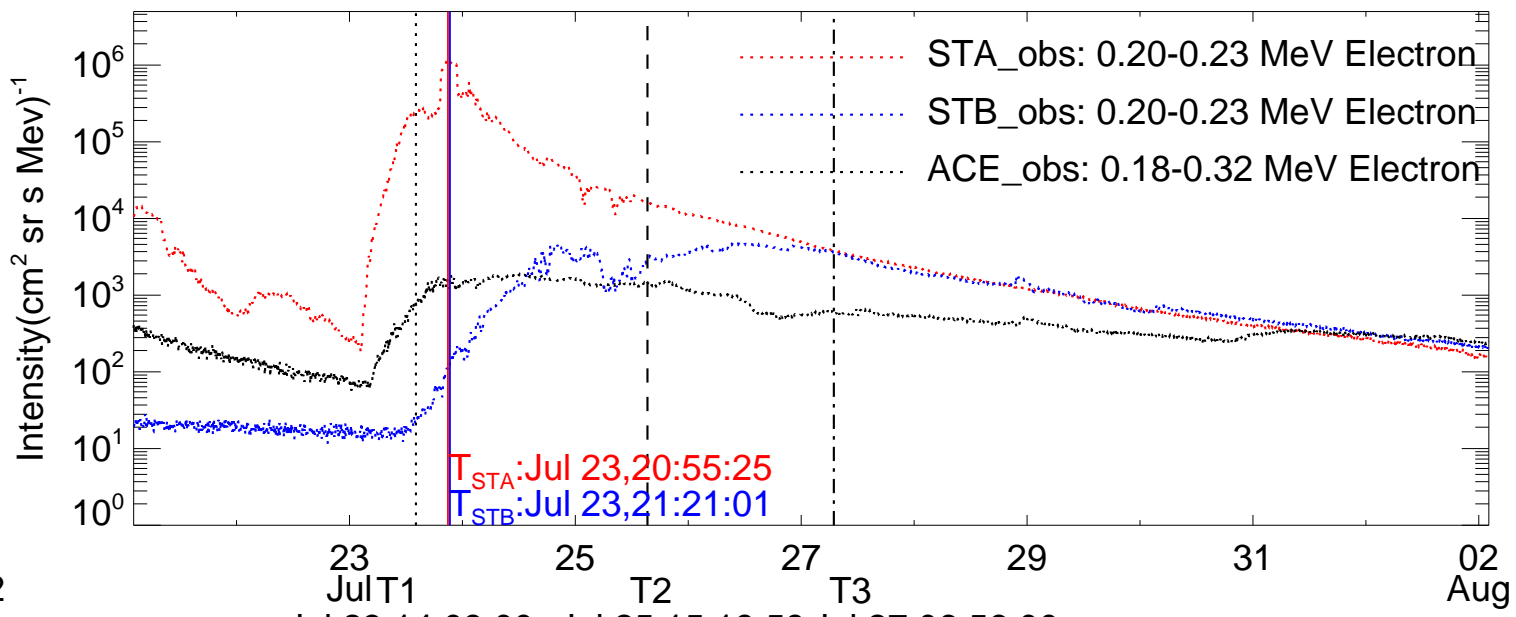

Jul 23,14:08:00 Jul 25,15:19:59 Jul 27,06:56:00

Fig. 3. Time profile of electron fluxes by the multi-spacecraft observation during the 23 July 2012 SEP event. The black, red, and blue dotted curves correspond to the observations of $0.18-0.32 \mathrm{MeV}$ electrons on ACE, and $0.2-0.23 \mathrm{MeV}$ electrons on STEREO-A and STEREO-B.

note that the SEP flux observation of STEREO-B began to rise around $T 1$ before the connection was actually established, one may assume that this is due to perpendicular diffusion of the energetic particles.

Third, we consider proton and electron observations by ACE with black curves in Figs. 2 and 3, respectively. Around T1, ACE observed an SEP intensity maximum for protons, and after about $8 \mathrm{~h}$ SEP intensity started to decrease (Fig. 2). However, for electrons, around $T 1$, ACE observed SEP intensity to continuously increase with time until it reached its maximum in a few hours and began to decrease after a few days (Fig. 3). Figure 4 shows that at $T 1$ the shock arrived at the position around $0.63 \mathrm{au}$. We can see that the cobpoint of ACE moved out from the edge of the shock, and so there was no connection between ACE and the shock. Therefore, one would expect a decrease of SEP intensity around that time rather than an intensity maximum. However, it is assumed that there was parallel diffusion, meaning that energetic particles were scattered back and forth along the magnetic field lines. Therefore, it is reasonable that the ACE proton SEP flux began to decrease several hours after $T 1$. However, the fact that the ACE electron SEP flux did not decrease until several days later is not easy to explain (see Fig. 5).

From Figs. 2 and 3 for protons and electrons, respectively, we also note that at the onset and peak time, the flux of SEPs measured by the three spacecraft, STEREO-A, STEREO-B, and ACE, se quite different. However, at the decay time, the electron SEP fluxes are at similar levels but the proton SEP fluxes are not. We assume electrons showed a "reservoir" phenomenon (McKibben 1972; Roelof et al. 1992; Qin et al. 2013; Wang \& Qin 2015).

\section{Solar energetic particle transport model}

In Sect. 2 we present a qualitative analysis of the propagation of SEPs accelerated by the interplanetary shock during the 23 July 2012 event. We then perform a quantitative study using the SEP transport model.

In this work, we use the shock particle transport code (Wang et al. 2012; Qin et al. 2013) later abbreviated to SPTC (Qi et al. 2017) to simulate the transport of the 23 July 2012 SEP event 


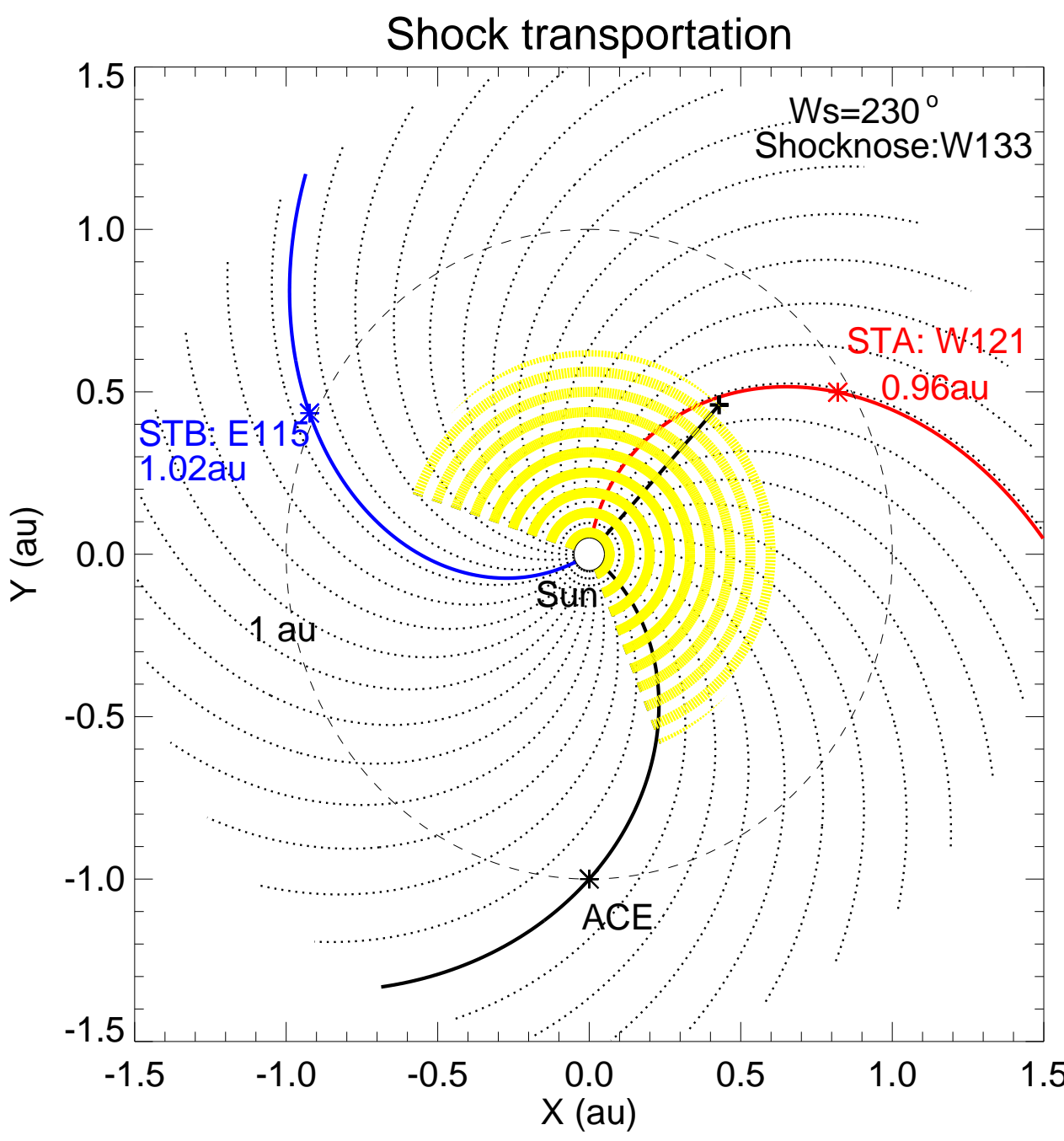

Fig. 4. Same as Fig. 1 except that the shock was at around 0.6 au on $T 1$, July 23, 14:08:00.

assuming the CME-driven shock to be a moving particle source. For the study of the transport mechanism of the SEPs (Qin et al. 2006; Zhang et al. 2009), we use the three-dimensional focused transport equation (Skilling 1971; Schlickeiser et al. 2002; Qin et al. 2006; Zhang et al. 2009):

$$
\begin{gathered}
\frac{\partial f}{\partial t}+\left(v \mu \hat{\mathbf{b}}+\mathbf{V}^{\mathrm{sw}}\right) \cdot \nabla f-\nabla \cdot\left(\kappa_{\perp} \cdot \nabla f\right)-\frac{\partial}{\partial \mu}\left(D_{\mu \mu} \frac{\partial f}{\partial \mu}\right) \\
-p\left[\frac{1-\mu^{2}}{2}\left(\nabla \cdot \mathbf{V}^{\mathrm{sw}}-\hat{\mathbf{b}} \hat{\mathbf{b}}: \nabla \boldsymbol{V}^{\mathrm{sw}}\right)+\mu^{2} \hat{\mathbf{b}} \hat{\mathbf{b}}: \nabla \mathbf{V}^{\mathrm{sw}}\right] \frac{\partial f}{\partial p} \\
+\frac{1-\mu^{2}}{2}\left[-\frac{v}{L}+\mu\left(\nabla \cdot \boldsymbol{V}^{\mathrm{sw}}-3 \hat{\mathbf{b}} \hat{\mathbf{b}}: \nabla \mathbf{V}^{\mathrm{sw}}\right)\right] \frac{\partial f}{\partial \mu}=0,
\end{gathered}
$$

where $f(\mathbf{x}, \mu, p, t)$ is the distribution function, $\mathbf{x}, \mu, p$ and $v$ are the particle position, pitch-angle cosine, momentum, and speed in a nonrotating heliographic coordinate system, respectively, and $t$ is time. Furthermore, $L=-B(s) /(\partial B / \partial s)$ is the magnetic focusing length. Equation (1) includes almost all important particle transport effects, such as particle streaming along field lines, adiabatic cooling in the expanding solar wind, perpendicular and pitch angle diffusion of particles, and magnetic focusing in the diverging interplanetary magnetic field (IMF) which is assumed as the Parker field. Here, $D_{\mu \mu}$ is the particle pitch-angle diffusion coefficient following Jokipii (1966), Teufel \& Schlickeriser (2003), and Burger et al. (2008). A quasi-linear model with the nonlinear effect of magnetic turbulence on the pitch angle scat- tering at $\mu=0$ (Qin \& Shalchi 2009) can be written as

$D_{\mu \mu}(\mu)=\frac{D_{\text {slab }} v}{l_{\text {slab }}}\left(\frac{R}{R_{\text {au }}}\right)^{-1 / 3}\left(|\mu|^{g-1}+h\right)\left(1-\mu^{2}\right)$.

The diffusion can be interpreted physically as being caused by the magnetic turbulence, where the spectral index in the inertial range $g=5 / 3$ is set according to the Kolmogorov index of the magnetic field turbulence power spectrum. The constant $h$ is set to 0.01 and $R=\mathrm{pc} /\left(|q| B_{0}\right)$ is the Larmor radius, $R_{\mathrm{au}}=1 \mathrm{au}$, and $q$ is the particle charge. Here, $l_{\text {slab }}$ is the slab turbulence correlation length which is set as $l_{\text {mslab }}=0.03 \mathrm{au}$. The relationship between the particle pitch angle diffusion coefficient and the parallel particle mean free path is (Jokipii 1966; Hasselmann \& Wibberenz 1968; Earl 1974)

$\lambda_{\|}=\frac{3}{8} v \int_{-1}^{1} \frac{\left(1-\mu^{2}\right)^{2}}{D_{\mu \mu}} \mathrm{d} \mu$,

and the parallel diffusion coefficient $\kappa_{\|}$can be written as $\kappa_{\|}=$ $v \lambda_{\|} / 3$.

Matthaeus et al. (2003) developed nonlinear guiding center (NLGC) theory for perpendicular diffusion, which was approximated in analytic form (Shalchi et al. 2004, 2010) for particles in certain parameter and energy ranges,

$\kappa_{\perp}=\frac{1}{3} v D_{2 D} l_{2 D}^{2 / 3} \times \lambda_{\|}^{1 / 3}(\mathbf{I}-\hat{\mathbf{b}} \hat{\mathbf{b}})$, 


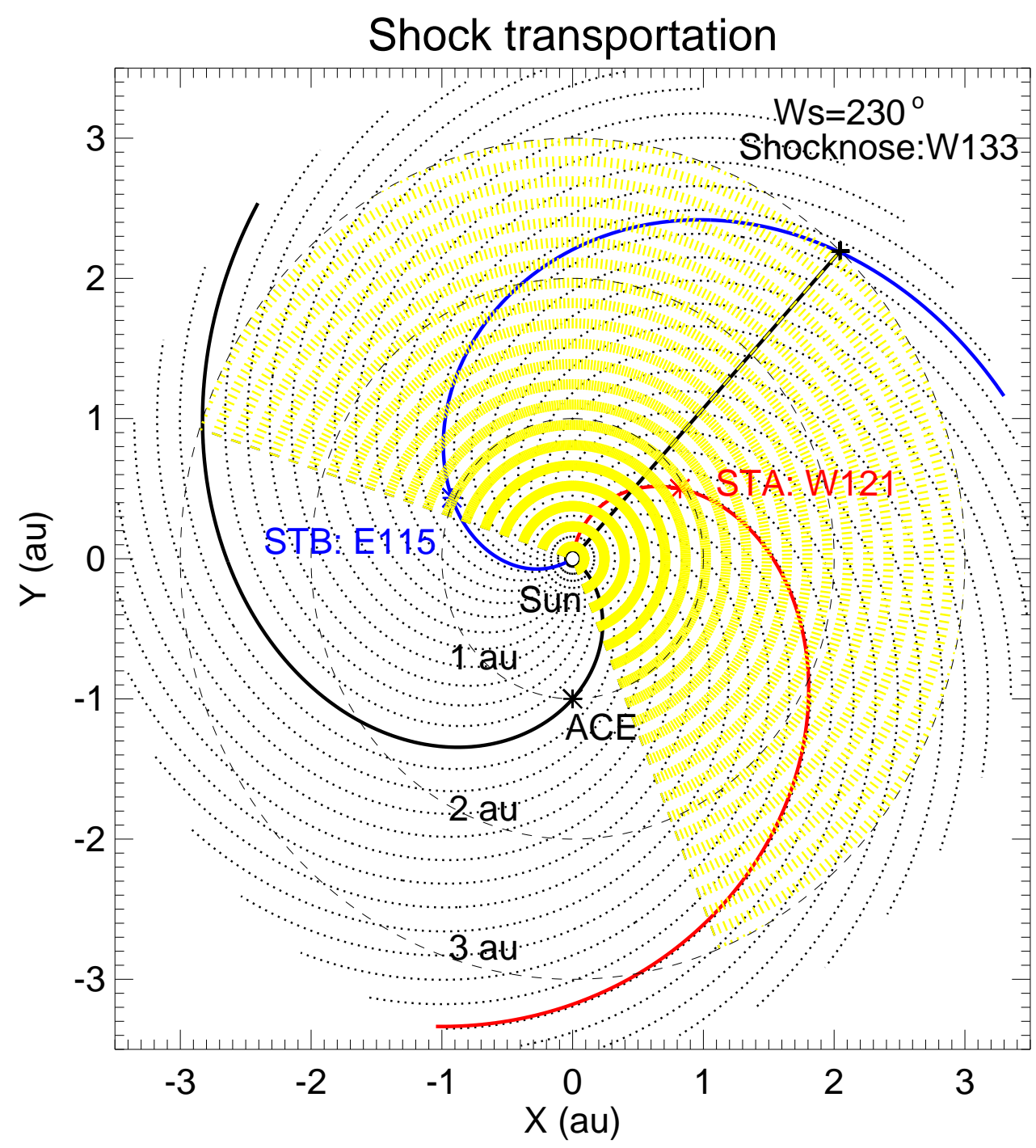

Fig. 5. Same as Fig. 1 except that the shock was at around 3 au on $T 2$, July 25 , 15:19:59. where $\mathbf{I}$ is a unit tensor, and $\kappa_{\perp}$ is set to be independent of $\mu$ due to the fact that particle pitch-angle diffusion is always much faster than perpendicular diffusion. According to research based on multi-spacecraft measurements (Weygnad et al. 2009, 2011), for the slow solar wind, we set the correlation scale ratio of the slab to $2 \mathrm{D}, l_{2 D} / l_{\text {slab }}=2.5$, so that $l_{2 D}=0.012 \mathrm{au}$. The perpendicular mean free path can be defined as $\lambda_{\perp} \equiv 3 \kappa_{\perp} / v$. Here, $D_{2 D}$ is a parameter that depends on the spectral index in the inertial range and the $2 \mathrm{D}$ component of the magnetic turbulence.

The above pitch angle diffusion model (2) and perpendicular diffusion model (4) are all in the analytic form to be used in our numerical simulation. However, according to previous work (Bieber et al. 1994, 2004), proton and electron mean free paths should have different model formats since they are fundamentally different. Therefore, the pitch angle diffusion models for protons and electrons should be different. To deal with this fact, we follow Bieber et al. (1994, 2004) and study particles pitch angle diffusion coefficients considering the difference between the mass of a proton and an electron. In addition, it is necessary to approximately develop the analytic forms of particle pitch angle diffusion coefficients for protons and electrons separately. However, this is beyond the scope of this study. For simplicity, we use the pitch angle diffusion model of Eq. (2) and the approximated analytic form of NLGC for perpendicular diffu- sion of Eq. (4) for both protons and electrons, but we assume the constant $D_{\text {slab }}$ for protons for any SEP event; $D_{\text {slab }}^{\mathrm{p}}$ is different from that for electrons $D_{\text {slab }}^{\mathrm{e}}$, and the constant $D_{2 D}$ for protons, $D_{2 D}^{\mathrm{p}}$, is different from that for electrons $D_{2 D}^{\mathrm{e}}$. The values of $D_{\text {slab}}^{\mathrm{p}}$, $D_{\text {slab }}^{\mathrm{e}}, D_{2 D}^{\mathrm{p}}$, and $D_{2 D}^{\mathrm{e}}$ of an SEP event can be obtained with diffusion theories of electrons and protons considering the nonlinear effects (e.g., Matthaeus et al. 2003; Bieber et al. 2004; Shalchi et al. 2004; Qin 2007) with parameters of magnetic turbulence in solar wind as input. However, theories for particle diffusion are still in the research and development stage, and details of solar wind magnetic turbulence are not available from observations. We therefore take the parameters of $D_{\text {slab }}^{\mathrm{p}}, D_{\text {slab }}^{\mathrm{e}}, D_{2 D}^{\mathrm{p}}$, and $D_{2 D}^{\mathrm{e}}$ to be constants for an SEP event.

Our model assumes the shock to be the source of particle injection with the boundary condition (Kallenrode \& Wibberenz 1997):

$f_{b}=a \delta\left(r-v_{\mathrm{s}} t\right)\left(\frac{r}{r_{c}}\right)^{\alpha} \exp \left[-\frac{|\phi(\theta, \varphi)|}{\phi_{c}(p)}\right] p^{\gamma} \xi(\theta, \varphi)$,

where the shock acceleration efficiency parameter $\alpha$ measures the damping rate with radial distance, and the other shock acceleration strength parameter $\phi_{c}$ describes the injection decrease from the middle to the flank of the shock. Here, $\gamma$ is the powerlaw spectrum index of the shock, and $\xi(\theta, \phi)$ shows the angular 
Table 1. Model parameters independent of the particle species used in the calculations.

\begin{tabular}{lcc}
\hline \hline Parameter & Physical meaning & Value \\
\hline$v_{\mathrm{s}}$ & Shock speed & $2003 \mathrm{~km} \mathrm{~s}^{-1}$ \\
$W_{\mathrm{s}}$ & Shock width & $230^{\circ}$ \\
$V^{\mathrm{SW}}$ & Solar wind speed & $450 \mathrm{~km} \mathrm{~s}^{-1}$ \\
$r_{\mathrm{O}}$ & Observer solar distance & $1 \mathrm{au}$ \\
$\Delta r$ & Shock space interval between two fresh injections & $0.001 \mathrm{au}$ \\
$r_{c}$ & Radial normalization parameter & $0.05 \mathrm{au}$ \\
$r_{b 0}$ & Inner boundary & $0.05 \mathrm{au}$ \\
$r_{b 1}$ & Outer boundary & $50 \mathrm{au}$ \\
$D_{\text {slab }}^{\mathrm{p}}$ & Constant for proton parallel diffusion & 0.063 \\
$D_{\mathrm{slab}}^{\mathrm{e}}$ & Constant for electron parallel diffusion & 0.0252 \\
$D_{2 D}^{\mathrm{p}}$ & Constant for proton perpendicular diffusion & 0.3 \\
$D_{2 D}^{\mathrm{e}}$ & Constant for electron perpendicular diffusion & 0.402 \\
\hline
\end{tabular}

range of the shock front (Wang et al. 2012),

$\xi(\theta, \varphi)= \begin{cases}1 & \text { if }|\phi(\theta, \varphi)| \leqslant \phi_{\mathrm{s}} \\ 0 & \text { otherwise }\end{cases}$

where $\phi(\theta, \varphi)$ is the angle between any particle injection position at the shock front and shock nose, and $\phi_{\mathrm{s}}$ is the half angular width of the shock.

We reformulate the transport Eq. (1) in terms of a set of stochastic differential equations, and solve it with a timebackward Markov stochastic process method using the Monte Carlo simulation (Zhang 1999). The particles are traced from the observation time back to the initial time of the injection at the source (Qin et al. 2006).

\section{Simulations and comparisons with observations}

In SPTC, the speed $v_{\mathrm{s}}$ and width $W_{\mathrm{s}}$ of the shock are input parameters. According to observations of the 23 July 2012 event, shock speed $v_{\mathrm{s}}$ is set as a constant $2000 \mathrm{~km} \mathrm{~s}^{-1}$. The shock width $W_{\mathrm{s}}$ is set as $230^{\circ}$ on account of the analysis of the possible shock width with the observation data discussed in Sect. 2 and shown in Fig. 1.

In order to solve the transport equation, we have to set the model parameters to some certain values. We compare the modeled outputs, that is, the simulation results, with the observational data by eye to get the best-fitting parameters. To fit the numerical simulations with the spacecraft observations of the 23 July 2012 event, we use the constant diffusion coefficients $D_{\text {slab }}^{\mathrm{p}}=0.0630, D_{\text {slab }}^{\mathrm{e}}=0.0252, D_{2 D}^{\mathrm{p}}=0.300$, and $D_{2 D}^{\mathrm{e}}=0.402$, from which we also obtain the ratio of diffusion coefficients between perpendicular and parallel diffusion: $4.4 \%$ and $10 \%$, for protons and electrons, respectively. In this work, all the simulation parameters independent of the particle species for the SEP event are shown in Table 1, and all the parameters that depend on particle species for the SEP event are listed in Table 2. The parameter values listed in Table 2 are the ones we obtained that would generate the optimal fitting results.

The shock strength parameter $\phi_{c}$ controls how fast the shock acceleration efficiency is reduced as the acceleration site changes from the nose to the edge. In addition, the shock acceleration efficiency parameter $\alpha$ controls how fast the acceleration efficiency of the shock changes while it propagates radially. As the CME of the 23 July 2012 event moved very quickly in the interplanetary space, the acceleration efficiency of the shock nose is much higher than that of the edge, and the parameter $\phi_{c}$ is very small.
Table 2. Model parameters depending on particle species used in simulations.

\begin{tabular}{lcccccc}
\hline \hline $\begin{array}{l}\text { Particle } \\
\text { Type }\end{array}$ & $\begin{array}{c}\text { Energy } \\
(\mathrm{MeV})\end{array}$ & $\alpha$ & $\phi_{c}$ & $\gamma$ & $\begin{array}{c}\lambda_{\|}{ }^{(1)} \\
(\mathrm{au})\end{array}$ & $\kappa_{\perp} / \kappa_{\|}{ }^{(1)}$ \\
\hline Protons & 3 & -2 & 3 & -2.5 & 0.18 & $4.4 \%$ \\
Electrons & 0.2 & -3 & 2 & -6.5 & 0.082 & $10 \%$ \\
\hline
\end{tabular}

Notes. ${ }^{(1)}$ For particles in the ecliptic at 1 au.

Moreover, the acceleration efficiency of the shock changed very quickly while it propagated radially, so $\alpha$ is relatively small.

From STEREO/SEPT we choose the proton channel with the energy range 2.2-6.5 MeV, and from ACE/EPAM we choose the proton channel with the energy range $1.91-4.75 \mathrm{MeV}$. We use $3 \mathrm{MeV}$ as the typical value for simulations to compare with the three observational proton channels. For each simulated data point of energetic particle flux, $2.88 \times 10^{7}$ pseudo-particles are used. The observation and simulation results of the time profile of SEP fluxes are shown in Fig. 6. The dotted and solid lines indicate observations and simulations, respectively. The black, red, and blue lines correspond to ACE, STEREO-A, and STEREOB, respectively. From 00:00 on 21 July to 00:00 on 23 July, we fit observational results with a straight line with intensity in log scale. We use the fitting results as the background for the simulation results for the whole time period, from 00:00 on 21 July to 00:00 on 2 August. We adjust the free parameter for the shock strength to get the best fit between observations and simulations.

From Fig. 6 we can see that the simulations and observations generally agree well for the three spacecraft. Particularly in terms of the timing for the start and peak of SEP flux, the simulations approximately agree with observations. However, there are differences in the details between the SEP flux of observations and simulations. We can see that for the SEP flux obtained by STEREO-A the observations have a sharper peak and a lower tail than simulations. This might be because there is no direct shock connection downstream of the shock. Specifically, when the shock reaches STEREO-A there is no significant increase in the simulation results. In addition, for the SEP flux observed by STEREO-B the observations have lower peak and tail than the simulations. Furthermore, for the SEP flux observed by ACE, the peak value of observations is larger than that of the simulations, but the tails of observations are lower than that of the simulations. We suppose that the spikes in the simulation results early 


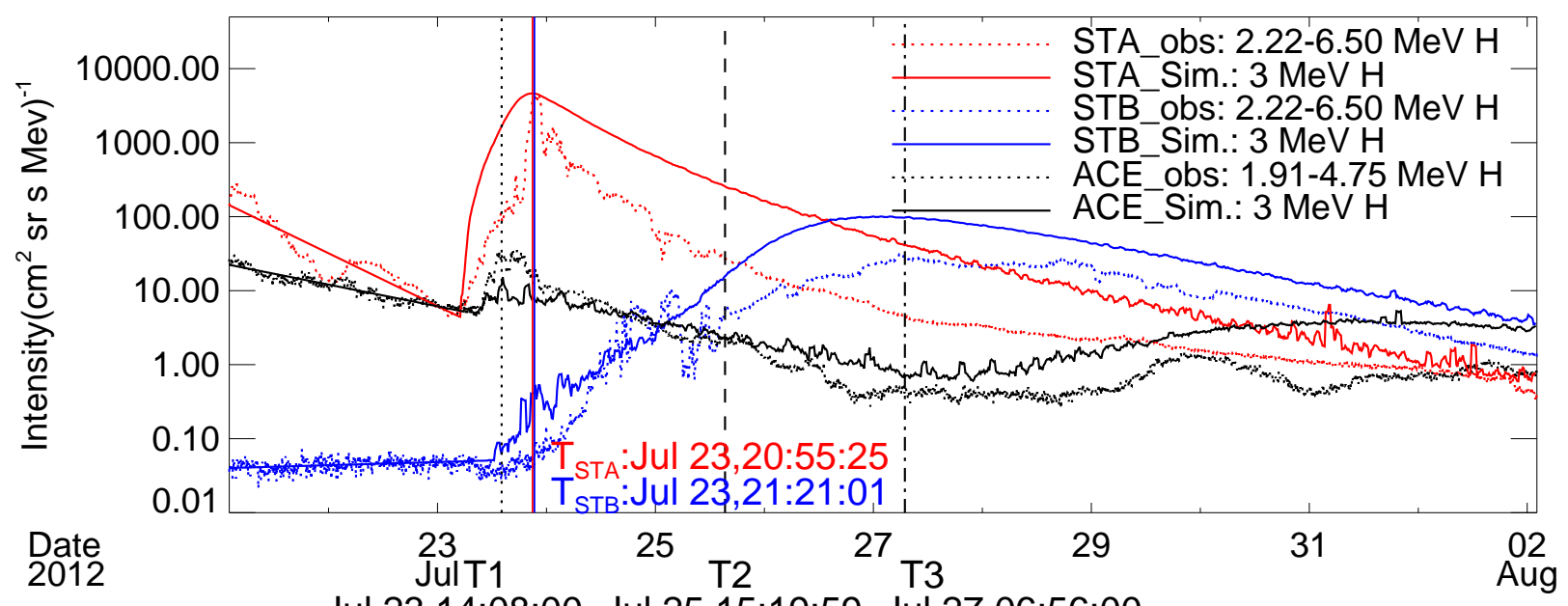

Jul 23,14:08:00 Jul 25,15:19:59 Jul 27,06:56:00

Fig. 6. Proton fluxes of the observations and simulations during the 23 July 2012 SEP event. The dotted and solid lines indicate the observations and simulations, respectively. The black, red, and blue lines correspond to the ACE, STEREO-A, and STEREO-B, respectively.

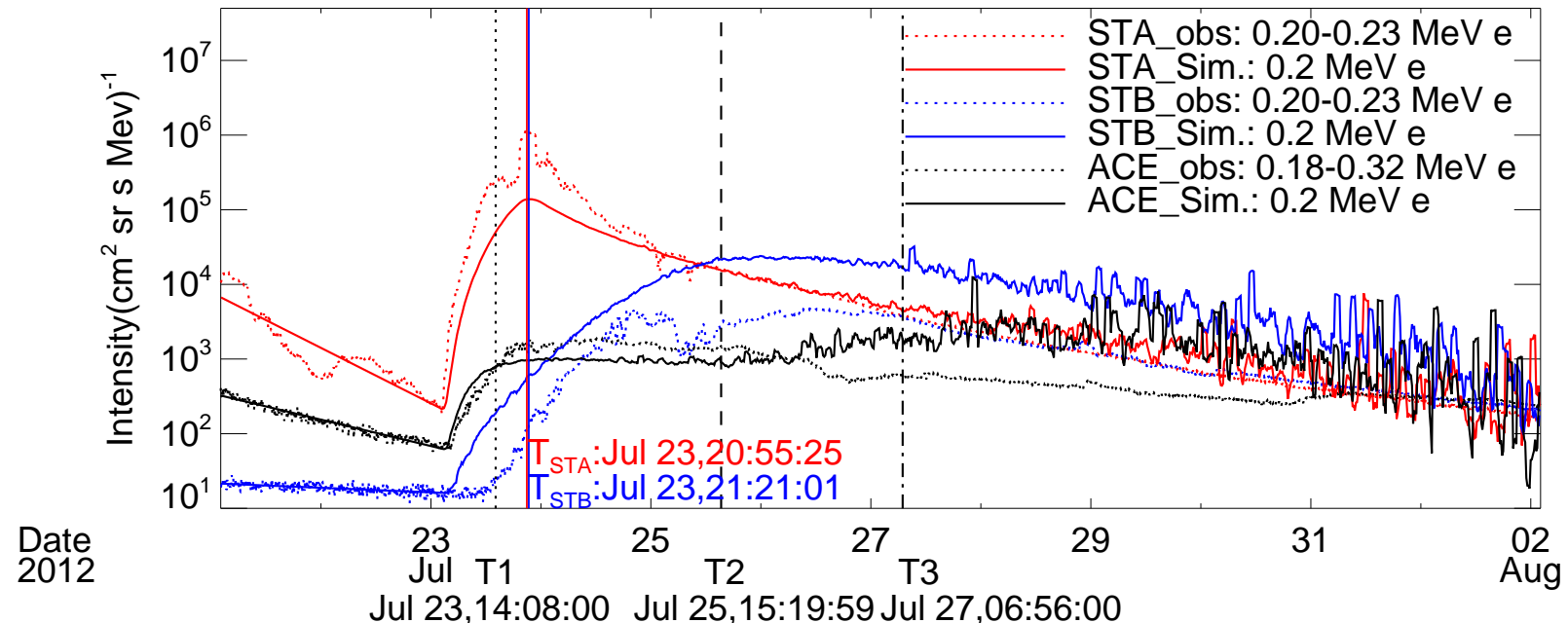

Fig. 7. Electron fluxes of the observations and simulations during the 23 July 2012 SEP event. The dotted and solid lines indicate the observations and simulations, respectively. The black, red, and blue lines correspond to the ACE, STEREO-A, and STEREO-B, respectively.

in the event are due to the low statistics of the simulation. It is noted that the background of the flux for STEREO-A and ACE before the SEP event dropped quickly from high levels due to the contribution of the previous CMEs which would have a large impact on STEREO-A and ACE. For the previous CMEs, there were three fast CME eruptions during the period from July 17 to 20 toward STEREO-A and ACE (Bain et al. 2016) ${ }^{4}$.

We also perform numerical simulations for $0.2 \mathrm{MeV}$ electrons with $1.091 \times 10^{7}$ pseudo-particles for each data point. In Fig. 7 we compare the simulation results with the electron observations of the EPAM instrument onboard ACE and the SEPT instruments onboard STEREO-A and STEREO-B. The ranges of energy we choose for the electron channel are $0.18-0.32 \mathrm{MeV}$ and $0.20-0.23 \mathrm{MeV}$ for EPAM and SEPT, respectively. From Fig. 7 we can see that the observations and simulations of STEREO-A are consistent except that the peak of observations is about one order of magnitude higher. As for protons, there is also no significant increase in the electron SEP flux from simulations when the shock reaches STEREO-A compared to the observa-

\footnotetext{
4 https://cdaw.gsfc.nasa.gov/CME_list/radio/waves_ type2.html
}

tions. On the other hand, the STEREO-B observations, with a later starting time than simulations, have a peak that is lower than the simulated one. Furthermore, the ACE observations and simulations agree relatively well. However, around $T 3$, there is an increase in the simulation of the flux as would be observed by ACE. We note that, similarly to the ACE protons early in the event, in the decay phase of the event, there are many spikes in the simulation results because of the poorer statistics in numerical calculations. In Table 3 we list the peak flux from observations and simulations for protons and electrons. It is noted that our model can only reproduce the general trend seen in the observational results, but the fitting of the peak is not good. From Figs. 6 and 7, it is shown that our simulations do not produce a reservoir satisfactorily. In SEP events, reservoir phenomena are usually observed during which particle intensities are nearly the same at different locations in decay phase (e.g., McKibben 1972; Roelof et al. 1992; Qin et al. 2013).

\section{Conclusion and discussion}

Here, we investigate the 23 July 2012 SEP event observed by multi-spacecraft STEREO-A, STEREO-B, and ACE, which 
Table 3. Peak values of observations and simulations.

\begin{tabular}{lccccc}
\hline \hline Particle & $\begin{array}{c}\text { Energy } \\
(\mathrm{MeV})\end{array}$ & $\begin{array}{c}\text { Data } \\
\text { Type }\end{array}$ & $\begin{array}{c}\text { STEREO-A } \\
\left(\mathrm{cm}^{2} \mathrm{sr} \mathrm{s} \mathrm{Mev}\right)^{-1}\end{array}$ & $\begin{array}{c}\text { STEREO-B } \\
\left(\mathrm{cm}^{2}{\mathrm{sr} \mathrm{s} \mathrm{Mev})^{-1}}^{-1}\right.\end{array}$ & $\begin{array}{c}\text { ACE } \\
\left(\mathrm{cm}^{2} \mathrm{sr} \mathrm{s} \mathrm{Mev}\right)^{-1}\end{array}$ \\
\hline Protons & 3 & obs & $4.6 \times 10^{3}$ & $3.1 \times 10^{1}$ & $3.5 \times 10^{1}$ \\
& & sim & $4.7 \times 10^{3}$ & $1.1 \times 10^{2}$ & $2.2 \times 10^{1}$ \\
\hline Electrons & 0.2 & obs & $1.2 \times 10^{6}$ & $4.7 \times 10^{3}$ & $1.9 \times 10^{3}$ \\
& & sim & $1.4 \times 10^{5}$ & $8.5 \times 10^{4}$ & $1.9 \times 10^{3}$ \\
\hline
\end{tabular}

are near $1 \mathrm{au}$ ecliptic with longitude at the event launch time: $\mathrm{W} 121.3^{\circ}, \mathrm{E} 115^{\circ}$, and $0^{\circ}$, respectively. The SEP event, which lasted for 10 days, was associated with ICME launched from the sunspot group NOAA $11520\left(\mathrm{~S} 15^{\circ} \mathrm{W} 133^{\circ}\right)$ at about $0208 \pm 2 \mathrm{~min}$ UT. However, this event is very complex. A wide variety of SEP anisotropies were observed. For example, bidirectional flows within the CMEs, sunward-flowing particles, and loss-cone distributions (Leske et al. 2015). In addition, there were some preceding CMEs during the period from July 17 to 20 (Bain et al. 2016; Zhu et al. 2016), but it is not known whether the observing spacecraft was connected to the preceding CMEs via magnetic field lines (Cohen et al. 2017). However, for the sake of simplicity, we still use the ideal simple model. We assume the shock nose direction is in the flare direction, $\mathrm{W} 133^{\circ}$. As the shock reached STEREO-A and STEREO-B but did not reach ACE, we assume a shock width of $W_{\mathrm{s}}=230^{\circ}$. For simplicity, the shock velocity is assumed to be constant in all directions.

First, we used the Parker's spiral magnetic field model to qualitatively analyze the relationship between the propagation of the CME-driven shock in the interplanetary space and the associated SEP flux observed by the multiple spacecraft. We provide a schematic to show their relative positional relationship. As the parallel diffusion of energetic particles is generally much larger than the perpendicular diffusion, for a qualitative analysis, we only consider the former. Our analysis is able to qualitatively explain some of the important features, especially in terms of the timing for the start and peak of SEP flux observed by STEREOA, STEREO-B, and ACE, simultaneously.

We then simulated the SEP event using the threedimensional focused transport model, by treating the shock as a moving energetic particle source. In the simulations, almost all the important particle transport effects, such as solar wind convection, particle streaming along the magnetic field line, magnetic focusing, adiabatic cooling, and the diffusion coefficients parallel and perpendicular to the IMF, are included. The simulations and observations approximately agree for the three spacecraft, especially in terms of the timing for the start and peak of SEP flux. Further, the schematic can qualitatively describe some characteristics of simulations and observations. However, there are differences in the details of the SEP flux of observations and simulations. Our modeling results only approximate follow the same trend as the observational results, but the fitting of the peak and onset is not satisfactory for STEREO-B, for example.

From observation results we can see the reservoir phenomenon for electrons, but we do not see this phenomenon for protons. A possible explanation of the phenomenon might be perpendicular diffusion (McKibben 1972; McKibben et al. 2001; Zhang et al. 2009; Qin et al. 2013) and magnetic boundaries (e.g., Reames et al. 1996). Furthermore, Wang \& Qin (2015) suggest that shock acceleration strength, parallel diffusion, and adiabatic cooling might be more important than perpendicular diffusion in forming the reservoir phenomenon. In our SEP mod- eling, we include all of the above effects, but in our simulations we do not produce a reservoir satisfactorily.

Proton and electron diffusion coefficients are fundamentally different (e.g., Bieber et al. 1994, 2004). However, for simplicity we still use the same models of the pitch angle diffusion from Eq. (2) and the approximated analytic form of NLGC for perpendicular diffusion from Eq. (4), but with different model parameters for protons and electrons to deal with their significant differences. In the future, it would be very interesting to develop increasingly realistic analytic forms of diffusion models for protons and electrons, respectively, for use in the simulation of transport and acceleration.

In the numerical model we consider shock as a moving SEP source without including physical acceleration processes when particles cross the shock. Therefore, in our simulation, when a shock reaches an observer there is no significant increase of flux compared to the observations. In the future, we will include the shock acceleration process (e.g., Kong et al. 2017; Qin et al. 2018 ) in our modeling work to make it more realistic.

In our model we used $450 \mathrm{~km} \mathrm{~s}^{-1}$ as a global value for solar wind speed $v_{\mathrm{sw}}$, which is a typical value measured by STEREO$\mathrm{A}$ and $-\mathrm{B}$ and $\mathrm{ACE}$ during the event. The solar wind speed is not the same in reality at different positions and times. For simplicity, we also assume that the shock speed $v_{\mathrm{s}}$ is constant throughout the interplanetary space in all directions. It is possible to use the mean value of the STEREO-A and -B shock measurements, $v_{\mathrm{sAB}}$, as $v_{\mathrm{s}}$. However, to take into consideration the fact that the shock speed would decrease when it propagates to places with a solar distance of more than $1 \mathrm{au}$, we use a constant shock speed of $v_{\mathrm{s}}=2000 \mathrm{~km} \mathrm{~s}^{-1}$, which is more than $10 \%$ smaller than $v_{\mathrm{sAB}}$. We attempted the same analysis with a higher shock speed and obtained qualitatively similar results. Since the speed of solar wind and shock in our model are not realistic, there are large effects leading to discrepancies between the model and observations. In addition, we assume a symmetric extent for the shock on both sides of the nose and a shock width of $W_{\mathrm{s}}=230^{\circ}$ so that the shock arrives at STEREO-B without arriving at ACE. Furthermore, the positions of the cobpoints for the different spacecraft at different times depend on the above choice. This chosen shock width and position have a large effect on the modeling results.

Energetic particles might be accelerated somewhere near the Sun, for example in the flare or the coronal shock. In future studies, we plan to include these mechanisms in our model. Furthermore, here, as is usually the case for transport modeling work, we assume a Parker model, that is, Archimedean spiral magnetic flux tubes in interplanetary space with constant solar wind speed measured in situ. However, the departure of the interplanetary magnetic field from the Parker model due to the impact of shock waves and ICME is not taken into account. Because we have adopted all the assumptions under such complicated conditions, our modeling results do not fit well enough to the observations. 
For example, the onset or the peak are not always well reproduced. However, our model is still valid because transport of the particles is significantly driven by the moving shock, which is connected to the observer by the magnetic field lines. In the future, we may use an MHD model to obtain more realistic settings for solar wind speed, magnetic field, shock speed, and so on.

Acknowledgements. We are partly supported by Grants NNSFC 41874206 and NNSFC 41574172. CME data are taken from the CDAW LASCO catalog, which is generated and maintained at the CDAW Data Center by NASA and The Catholic University of America in cooperation with the Naval Research Laboratory. The work was carried out at National Supercomputer Center in Tianjin, and the calculations were performed on TianHe-1 (A). We gratefully acknowledge Yang Wang, Shaw Qin, and Ying Qin for useful discussions about this topic.

\section{References}

Bain, H. M., Mays, M. L., Luhmann, J. G., et al. 2016, ApJ, 825, 1

Bieber, J. W., Matthaeus, W. H., Smith, C. W., et al. 1994, ApJ, 420, 294

Bieber, J. W., Matthaeus, W. H., Shalchi, A., \& Qin, G. 2004, Geophys. Res. Lett., 31, L10805

Beeck, J., \& Wibberenz, G. 1986, ApJ, 311, 437

Brueckner, G. E., Howard, R. A., Koomen, M. J., et al. 1995, Sol. Phys., 162, 357

Burger, R. A., Krüger, T. P. J., Hitge, M., \& Engelbrecht, N. E. 2008, ApJ, 674 511

Cane, H. V., Richardson, I. G., \& von Rosenvinge, T. T. 2010, J. Geophys. Res. 115, A08101

Cohen, C., Bain, H.M., Li, Y., et al. 2017, PoS, 134,

Dresing, N., Gómez-Herrero, R., Klassen, A., et al. 2012, Sol. Phys., 281, 281

Dresing, N., Theesen, S., Klassen, A., \& Heber, B. 2016, A\&A, 588, A17

Earl, J. A. 1974, ApJ, 193, 231

Gordon, B. E., Lee, M. A., Möbius, E., \& Trattner, K. J. 1999, J. Geophys. Res., 104,28263

Hasselmann, K., \& Wibberenz, G. 1968, Z. Geophys., 34, 353

Heras, A. M., Sanahuja, B., Smith, Z. K., Detman, T., \& Dryer, M. 1992, ApJ, 391,359

Heras, A. M., Sanahuja, B., Lario, D., et al. 1995, ApJ, 445, 497

Hu, J., Li, G., Fu, S., Zank, G., \& Ao, X. 2018, ApJ, 854, L19

Jokipii, J. R. 1966, ApJ, 146, 480

Joyce, C. J., Schwadron, N. A., Townsend, L. W., et al. 2015, Space Weather, 13 560

Kahler, S. W. 2013, ApJ, 769, 110

Kahler, S. W., \& Vourlidas, A. 2013, ApJ, 769, 143

Kallenrode, M.-B. 2001, J. Geophys. Res., 106, 24989

Kallenrode, M.-B., \& Wibberenz, G. 1997, J. Geophys. Res., 102, 22311

Kong, F.-J., Qin, G., \& Zhang, L.-H. 2017, ApJ, 845, 43

Lario, D., Sanahuja, B., \& Heras, A. M. 1998, ApJ, 509, 415

Lario, D., Ho, G. C., Decker, R. B., et al. 2003, AIP Conf. Proc., 679, 640

Lee, M. A. 1983, J. Geophys. Res., 88, 6109

Leske, R., Cummings, A., Cohen, C., et al. 2015, 34th Int. Cosmic Ray Conf., 236, 072

Li, G., Zank, G. P., \& Rice, W. K. M. 2003, J. Geophys. Res., 108, 1082
Liou, K., Wu, C.-C., Dryer, M., et al. 2014, J. Atm. Sol.-Terr. Phys., 121, 32 Liu, Y. D., Luhmann, J. G., Kajdič, P., et al. 2014, Nat. Commun., 5, 3481 Matthaeus, W. H., Qin, G., Bieber, J. W., \& Zank, G. P. 2003, ApJ, 590, 53 McKibben, R. B. 1972, J. Geophys. Res., 77, 3957

McKibben, R. B., Connell, J. J., Lopate, C., et al. 2001, Int. Cosmic Ray Conf., 8,3281

Ng, C. K., Reames, D. V., \& Tylka, A. J. 1999, Geophys. Res. Lett., 26, 2145

Pan, Z., Wang, C., Wang, Y., \& Xue, X. 2011, Sol. Phys., 270, 593

Qin, G. 2007, ApJ, 656, 217

Qi, S.-Y., Qin, G., \& Wang, Y. 2017, Res. Astron. Astrophys., 17, 33

Qin, G., \& Shalchi, A. 2009, ApJ, 707, 61

Qin, G., \& Shalchi, A. 2014a, Appl. Phys. Res., 6, 1

Qin, G., \& Shalchi, A. 2014b, Phys. Plasmas, 21, 042906 (1994-present)

Qin, G., \& Wang, Y. 2015, ApJ, 809, 177

Qin, G., \& Zhang, L.-H. 2014, ApJ, 787, 12

Qin, G., Matthaeus, W. H., \& Bieber, J. W. 2002a, Geophys. Res. Lett., 29, 1048

Qin, G., Matthaeus, W. H., \& Bieber, J. W. 2002b, ApJ, 578, L117

Qin, G., Zhang, M., \& Dwyer, J. R. 2006, ApJ, 111, 8101

Qin, G., Wang, Y., Zhang, M., \& Dalla, S. 2013, ApJ, 766, 74

Qin, G., Kong, F.-J., \& Zhang, L.-H. 2018, ApJ, 860, 3

Reames, D. V. 1995, Rev. Geophys. Suppl., 33, 585

Reames, D. V. 1999, Space Sci. Rev., 90, 413

Reames, D. V. 2013, Space Sci. Rev., 175, 53

Reames, D. V., Barbier, L. M., \& Ng, C. K. 1996, ApJ, 466, 473

Reinard, A. A., \& Andrews, M. A. 2006, Adv. Space Res., 38, 480

Rice, W. K. M., Zank, G. P., \& Li, G. 2003, J. Geophys. Res., 108, 1369

Riley, P., Caplan, R. M., Giacalone, J., Lario, D., \& Liu, Y. 2016, ApJ, 819, 57

Roelof, E. C., Gold, R. E., Simnett, G. M., et al. 1992, Geophys. Res. Lett., 19, 1243

Russell, C. T., Mewaldt, R. A., Luhmann, J. G., et al. 2013, ApJ, 770, 38

Schlickeiser, R. 2002, in Cosmic Ray Astrophysics, ed. R. Schlickeiser

Shalchi, A., Bieber, J. W., \& Matthaeus, W. H. 2004, ApJ, 604, 675

Shalchi, A., Li, G., \& Zank, G. P. 2010, Ap\&SS, 325, 99

Skilling, J. 1971, ApJ, 170, 265

Sokolov, I. V., Roussev, I. I., Gombosi, T. I., et al. 2004, ApJ, 616, L171

Tan, L. C., Reames, D. V., Ng, C. K., Saloniemi, O., \& Wang, L. 2009, ApJ, 701, 1753

Temmer, M., \& Nitta, N. V. 2015, Sol. Phys., 290, 919

Teufel, A., \& Schlickeriser, R. 2003, A\&A, 397, 15

von Rosenvinge, T. T., Barbier, L. M., Karsch, J., et al. 1995, Space Sci. Rev., 71,155

Wang, Y., \& Qin, G. 2015, ApJ, 806, 252

Wang, Y., Qin, G., \& Zhang, M. 2012, ApJ, 752, 37

Weygnad, J. M., Matthaeus, W. H., Dasso, S., et al. 2009, J. Geophys. Res., 114, A07213

Weygnad, J. M., Matthaeus, W. H., Dasso, S., \& Kivelson, M. G. 2011, J. Geophys. Res., 116, A08102

Wu, S.-S., \& Qin, G. 2018, J. Geophys. Res., 123, 76

Zank, G. P., Rice, W. K. M., \& Wu, C. C. 2000, J. Geophys. Res., 105, 25079

Zank, G., Li, G., Florinski, V., \& Matthaeus, W. H. 2004, J. Geophys. Res., 109, A04107

Zank, G., Li, G., Florinski, V., et al. 2006, J. Geophys. Res., 111, A06108

Zhang, M. 1999, ApJ, 513, 409

Zhang, M., Qin, G., \& Rassoul, H. 2009, ApJ, 692, 109

Zhang, J. J., Wang, C., Sun, T. R., \& Liu, Y. D. 2016, Space Weather, 14, 259

Zhu, B., Liu, Y. D., Luhmann, J. G., et al. 2016, ApJ, 827, 146

Zuo, P., Zhang, M., Gamayunov, K., Rassoul, H., \& Luo, X. 2011, ApJ, 738, 168

Zuo, P., Zhang, M., \& Rassoul, H. K. 2013, ApJ, 767, 6 